\title{
Comparing Pitch Spelling Algorithms on a Large Corpus of Tonal Music
}

\author{
David Meredith \\ Centre for Computational Creativity, City University, London, \\ Northampton Square, London, EC1V 0HB, United Kingdom \\ dave@titanmusic.com \\ http://www.titanmusic.com
}

\begin{abstract}
This paper focuses on the problem of constructing a reliable pitch spelling algorithm - that is, an algorithm that computes the correct pitch names (e.g., $\mathrm{C} \sharp 4, \mathrm{Bb} 5$ etc.) of the notes in a passage of tonal music, when given only the onset-time, MIDI note number and possibly the duration of each note. The author's ps13 algorithm and the pitch spelling algorithms of Cambouropoulos, Temperley and Longuet-Higgins were run on a corpus of tonal music containing 1.73 million notes. ps13 spelt significantly more of the notes in this corpus correctly than the other algorithms (99.33\% correct). However, Temperley's algorithm spelt significantly more intervals between consecutive notes correctly than the other algorithms $(99.45 \%$ correct). All the algorithms performed less well on classical music than baroque music. However, ps13 performed more consistently across the various composers and styles than the other algorithms.
\end{abstract}

\section{Introduction}

\subsection{The Concept of a Pitch Spelling Algorithm}

In this paper, I focus on the problem of constructing a reliable pitch spelling algorithm - that is, an algorithm that reliably computes the correct pitch names (e.g., $\mathrm{C} \sharp 4, \mathrm{~B} b 5$ etc.) of the notes in a passage of tonal music, when given only the onset-time, MIDI note number and possibly the duration of each note in the passage.

There are good practical and scientific reasons for attempting to develop a reliable pitch spelling algorithm. First, until such an algorithm is devised, it will be impossible to construct a reliable MIDI-to-notation transcription algorithmthat is, an algorithm that reliably computes a correctly notated score of a passage when given only a MIDI file of the passage as input. Second, existing audio transcription systems generate not notated scores but MIDI-like representations as output [1-3]. So, if one needs to produce a notated score from a digital audio recording, a MIDI-to-notation transcription algorithm (incorporating a pitch spelling algorithm) is required in addition to an audio transcription system.

Third, knowing the letter-names of the pitch events in a passage can be extremely useful in music information retrieval and musical pattern discovery [4]. 


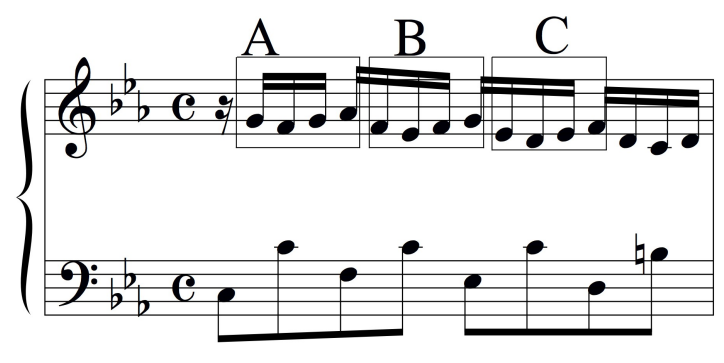

Fig. 1. Three perceptually similar patterns with different chromatic pitch interval structures (from the first bar of the Prelude in C minor (BWV 871/1) from Book 2 of J. S. Bach's Das Wohltemperirte Clavier)

In particular, two occurrences of a motive on different degrees of a scale might be perceptually similar even if the corresponding chromatic intervals in the patterns differ. For example, the three patterns A, B and C in Fig. 1 are perceived as being three occurrences of the same motive even though the corresponding chromatic intervals are different in the three patterns. Note that, in this example, one important aspect of the perceived similarity between patterns A, B and C is nicely represented in the notation by the fact that they all have the same scalestep interval structure (i.e., a downward step followed by two consecutive upward steps). In other words, one result of the choice of pitch names for the notes in this passage is that the scale-step interval structures are the same for these three perceptually similar but chromatically different patterns. This illustrates the fact that a correctly notated score is much more than simply a set of instructions for the performer (cf. tablature). A correct Western staff notation score of a passage of tonal music is a structural description of the piece that represents certain important aspects of the way that the piece is intended to be perceived and interpreted.

Matches such as the ones in Fig. 1 can be found using fast, exact-matching algorithms if the pitch names of the notes are encoded, but exact-matching algorithms cannot be used to find such matches if the pitches are represented using just MIDI note numbers. If a reliable pitch spelling algorithm existed, it could be used to compute the pitch names of the notes in the tens of thousands of MIDI files of works that are freely available online, allowing these files to be searched more effectively by a music information retrieval (MIR) system.

\subsection{Pitch Spelling In Common Practice Western Tonal Music}

In the vast majority of cases, the correct pitch name for a note in a passage of tonal music can be determined by considering the rôles that the note plays in the harmonic, motivic and voice-leading structures of the passage. For example, when played in isolation in an equal-tempered tuning system, the first soprano note in Fig. 2(a) would sound the same as the first soprano note in Fig. 2(b). 
However, in Fig. 2(a), this note is spelt as a $\mathrm{G} \sharp$ because it functions as a leading note in A minor; whereas in Fig. 2(b), the first soprano note is spelt as an Ab because it functions as a submediant in $\mathrm{C}$ minor. Similarly, the first alto note in Fig. 2(b) would sound the same as the first alto note in Fig. 2(c) in an equaltempered tuning system. However, in Fig. 2(b) the first alto note is spelt as an $\mathrm{F} t$ because it functions in this context as a subdominant in $\mathrm{C}$ minor; whereas, in Fig. 2(c), the first alto note functions as a leading note in $\mathrm{F} \sharp$ minor so it is spelt as an $E_{\sharp}$.

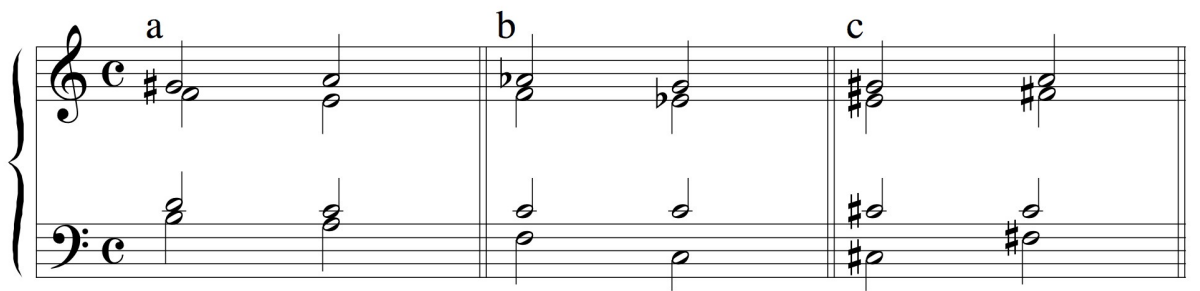

Fig. 2. Examples of enharmonic spelling (from [5, p. 8])

These examples illustrate that, in general, the pitch names assigned to notes in a passage of tonal music are not arbitrary. In most cases, they are carefully chosen so that the resulting score represents as well as possible certain important aspects of the way that the music is intended to be perceived and interpreted.

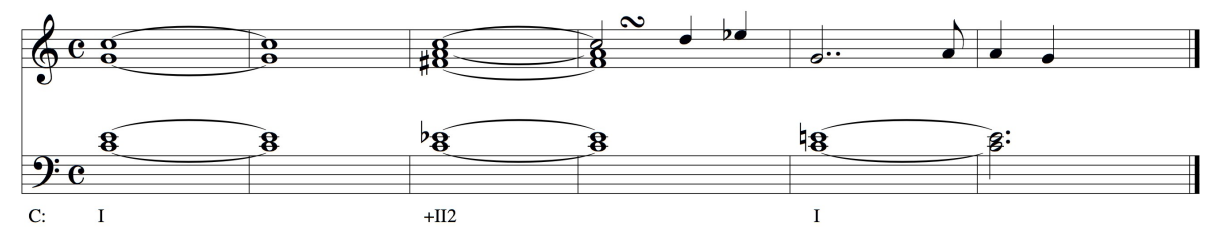

Fig. 3. Should the Ebs be spelt as $\mathrm{D} \sharp s$ ? (From [5, p. 390])

Of course, there do exist cases where it is difficult to determine the correct pitch name of a note uniquely by considering the harmonic, motivic and voiceleading structures of its context. For example, as Piston observes [5, p. 390], the tenor Eb4 in the third and fourth bars of Fig. 3 should be spelt as a $\mathrm{D} \sharp 4$ if one perceives the harmonic progression here to be ${ }^{+} \mathrm{II}^{2}-\mathrm{I}$ as proposed by Piston. But spelling the soprano Eb5 in the fourth bar as $\mathrm{D} \sharp 5$ would not represent the perceived structure of the melody correctly. 


\subsection{Using Accurate Encodings of Authoritative Editions as a 'Ground Truth'}

Fortunately, however, cases such as the one in Fig. 3 where it is difficult to determine the correct pitch name of a note are relatively rare - particularly in Western tonal music of the so-called 'common practice' period (roughly the 18th and 19th centuries). In the vast majority of cases, those who study and perform Western tonal music agree about how a note should be spelt in a given tonal context. Correspondingly, the vast majority of notes in authoritative published editions of scores of common practice tonal works are generally agreed to be spelt correctly by those who understand Western staff notation.

Therefore a pitch spelling algorithm can be evaluated quantitatively by running it on tonal works and comparing the pitch names it predicts with those of the corresponding notes in authoritative published editions of scores of the works. In other words, such authoritative scores can provide us with a 'ground truth' that we can compare with the output of a pitch spelling algorithm.

However, this can only be done accurately and quickly if one has access to accurate encodings of these authoritative scores in the form of computer files that can be compared automatically with the pitch spelling algorithm's output. Currently there exist only a small number of publicly available collections of encodings of authoritative editions of musical scores (e.g., the MuseData collection (http://www.musedata.org) and the Mutopia Project (http://www.mutopiaproject.org)). However, if real progress is to be made in the development and testing of systems for music analysis, retrieval and transcription, it is necessary for much larger and more varied corpora of encoded scores to be made publicly available (or at least available for research purposes). Moreover, it is necessary for these corpora to be highly accurate. Unfortunately, building such corpora is currently an extremely time-consuming and error-prone process despite the existence of, for example, optical music recognition (OMR) systems (for an overview, see David Bainbridge's web page at

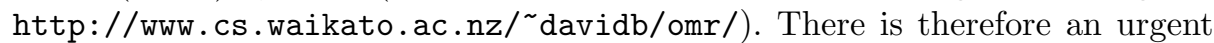
need for tools and techniques that will allow notated scores to be encoded more quickly and more accurately.

\section{A Comparison of Three Pitch Spelling Algorithms}

\subsection{Introduction}

In order to get a clearer idea of the 'state of the art' in the field, three pitch spelling algorithms were run on two test corpora and their performance was compared. The algorithms compared were those of Cambouropoulos [6-9], LonguetHiggins [10-12] and Temperley [13,14]. In an initial pilot study [15], the corpus used was the first book of J. S. Bach's Das Wohltemperirte Clavier ${ }^{1}$ (BWV 846869), which contains 41544 notes. Then a larger-scale comparison was carried

\footnotetext{
${ }^{1}$ This is Bach's own spelling of the work's title.
} 
out using a corpus containing 1729886 notes and consisting of 1655 movements from works by 9 baroque and classical composers (Corelli, Vivaldi, Telemann, Bach, Handel, B. Marcello, Haydn, Mozart and Beethoven). Both corpora were derived from the MuseData collection of encoded scores [16].

\subsection{Longuet-Higgins's Algorithm}

Pitch spelling is one of the tasks performed by Longuet-Higgins's music.p program [10-12]. The input to music.p must be in the form of a list of triples, $\left\langle p, t_{\mathrm{On}}, t_{\mathrm{off}}\right\rangle$, each triple giving the "keyboard position" $p$ together with the onset time $t_{\text {On }}$ and the offset time $t_{\text {off }}$ in centiseconds of each note. The keyboard position $p$ is just 48 less than the MIDI note number. So, for example, the keyboard position of middle $\mathrm{C}$ is 12 . Longuet-Higgins intended the music . p program to be used only on monophonic melodies and explicitly warns against using it on "accompanied melodies" or what he calls "covertly polyphonic" melodies (i.e., compound melodies) [11, p. 114].

It is perhaps also worth pointing out that the pitch spelling algorithm implemented in music.p does not use Longuet-Higgins's well-known threedimensional 'tonal space' model of tonality [11, p. 110-111]. However, LonguetHiggins does actually describe this multi-dimensional model in the paper where the music.p program is published. This has probably led some readers to assume (incorrectly) that the program implements Longuet-Higgins's three-dimensional 'tonal space' model. In fact, the only pitch spaces used in the pitch spelling algorithm implemented in music.p are the circle of fifths and the 'line of fifths' (see Fig. 4).

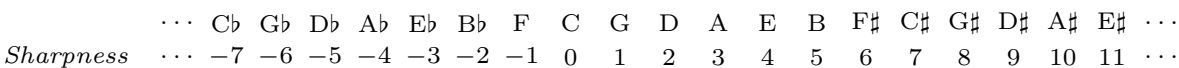

Fig. 4. The line of fifths

The algorithm computes a value of "sharpness" for each note in the input [11, p. 111]. The sharpness of a note is a number indicating the position of the pitch name of the note on the line of fifths (see Fig. 4) [14, p. 117]. It is therefore essentially the same as Temperley's concept of tonal pitch class [14, p. 118] and Regener's concept of quint [17, p. 33].

Longuet-Higgins's algorithm tries to spell the notes so that they are as close as possible to the local tonic on the line of fifths [11, pp. 112-113]. The algorithm assumes at the beginning of the music that the first note is either the tonic or the dominant of the opening key and chooses between these two possibilities on the basis of the interval between the first two notes [11, p. 114]. The algorithm also disallows consecutive chromatic intervals [11, p. 113] and incorporates a special rule for dealing with ascending semitones [11, p. 114]. 


\subsection{Cambouropoulos's Algorithm}

The input to Cambouropoulos's method [6-9] is again a sequence of MIDI note numbers in the order in which they occur in the music. The algorithm uses a "shifting overlapping windowing technique" [9, p. 420], as illustrated in Fig. 5, in which each window contains a certain number (typically 9 or 12) of contiguous elements in the input sequence.

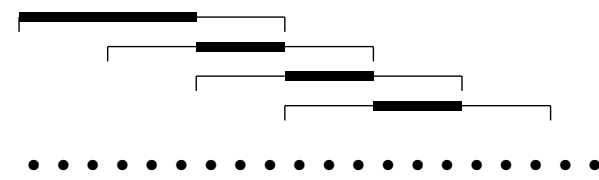

Fig. 5. Cambouropoulos's own caption to this figure reads: "Shifting overlapping window technique. For each window only the middle section of spelled pitches (bold line) is retained. Dots represent the pitches of the input sequence." (Reproduced (with minor corrections) from [9, p. 420, Fig. 6].)

On each step, the window position advances by a third of the window size, as shown in Fig. 5. Windowing improves the running time of the algorithm (from exponential to linear in the size of the input) and overlapping the windows avoids certain types of errors at window boundaries.

Cambouropoulos allows 'white note' pitch classes (i.e., 0, 2, 4, 5, 7, 9 and 11) to be spelt in three ways. For example, pitch class 0 can be spelt as B, C $\mathrm{C}$ or Dbb. 'Black note' pitch classes can be spelt in two ways. For example, pitch class 6 can be spelt as F\# or Gb (see, for example, [6, p. 242]). Given these restricted pitch name possibilities for each note, Cambouropoulos's method computes all the spellings for each window that do not contain both double-sharps and doubleflats. For example, for a 9-note window, 128 different spellings will have to be evaluated.

A penalty score is then computed for each of these possible window spellings. The penalty score for a given window spelling is found by computing a penalty value for each pitch interval in the window and summing these interval penalty values. A given interval in a particular window spelling is penalised more heavily the less frequently it occurs in the major and minor scales, a principle that Cambouropoulos calls interval optimization [9, p. 421]. An interval is also penalised if either of the pitch names forming the interval is a double-sharp or a double-flat, a principle that Cambouropoulos calls notational parsimony [9, p. 421]. For each window, the algorithm chooses the spelling that has the lowest penalty score and retains the pitch names for the middle third of this best window spelling.

\subsection{Temperley's Algorithm}

Temperley's pitch spelling algorithm [13,14] is implemented in his harmony program which forms one component of his and Sleator's Melisma system. The 
input to the harmony program must be in the form of a "note-list" [14, pp. 9-12] giving the MIDI note number of each note together with its onset time and duration in milliseconds. This note list must be accompanied by a representation of the metrical structure of the passage in the form of a "beat-list" of the type generated by Temperley and Sleator's meter program.

Temperley's pitch spelling algorithm searches for the spelling that best satisfies three "preference rules" [14, pp. 115-136]. The first of these rules stipulates that the algorithm should "prefer to label nearby events so that they are close together on the line of fifths" [14, p. 125]. This rule bears some resemblance to the basic principle underlying Longuet-Higgins's algorithm (see above). The second rule expresses the principle that if two tones are separated by a semitone and the first tone is distant from the key centre, then the interval between them should preferably be spelt as a diatonic semitone rather than a chromatic one [14, p. 129]. This rule is also very similar to one of the rules used in Longuet-Higgins's algorithm. The third preference rule steers the algorithm towards spelling the notes so that what Temperley calls a "good harmonic representation" results $[14$, p. 131].

Note, however, that Temperley's algorithm requires more information in its input than the other algorithms. In particular, it needs to know the duration of each note and the tempo at each point in the passage. It also needs to perform a full analysis of the metrical and harmonic structure of the passage in order to generate a high quality result. Also, it cannot deal with cases where two or more notes with the same pitch start at the same time.

\subsection{Results of Running Algorithms on the First Book of J. S. Bach's Das Wohltemperirte Clavier}

Table 1 shows the results obtained in a pilot study in which the three algorithms described above were run on the first book of J. S. Bach's Das Wohltemperirte Clavier [15]. As can be seen, Temperley's algorithm performed best, followed by Longuet-Higgins's algorithm and then Cambouropoulos's.

Table 1. Results obtained when the three algorithms were run on the first book of J. S. Bach's Das Wohltemperirte Clavier

\begin{tabular}{|c|c|c|}
\hline Algorithm & $\%$ notes correct & Number of errors \\
\hline Cambouropoulos & 93.74 & 2599 \\
Longuet-Higgins & 99.36 & 265 \\
Temperley & 99.71 & 122 \\
\hline
\end{tabular}

Total number of notes in corpus $=41544$.

As mentioned above, Longuet-Higgins's algorithm was not designed to be used on polyphonic music. Therefore, before running this algorithm on the corpus, each piece was first represented as a sequence of MIDI note numbers in the 
order in which they occur in the music. Within each set of note numbers corresponding to a set of notes beginning simultaneously, the elements were sorted into increasing order by note number.

\section{The ps13 Algorithm}

\subsection{Description of the Algorithm}

Having carried out this pilot study and gained a better idea of the 'state of the art' in the field, an attempt was made to construct a new algorithm that improved on Temperley's. Around 30 different algorithms were developed and tested and the one that performed best on the first book of Bach's Das Wohltemperirte Clavier will now be described. This algorithm is called ps13. $^{2}$

The input to $p s 13$ is, again, a sequence of MIDI note numbers in which the ordering corresponds to the order in which the notes occur in the music, any set of note numbers corresponding to notes that begin simultaneously being sorted into increasing order by note number. This sequence of MIDI note numbers is then converted into a sequence of pitch classes (if the MIDI note number of a note is $n$, then the pitch class of the note is $n \bmod 12)$. At the highest level of description, ps13 can be broken down into two stages, which I shall call Stage 1 and Stage 2. In the following description, I denote by $C$ the ordered set of pitch classes given to the algorithm as input. $C[i]$ denotes the $(i+1)$ th element of $C$ (e.g., $C[0]$ is the first element in $C$ ) and $|C|$ denotes the length of the sequence $C$.

Stage 1 involves carrying out the following steps:

Step 1 For each $0 \leq i<|C|$ and each pitch class $0 \leq p \leq 11$, compute a value $C N T(i, p)$ giving the number of times that $p$ occurs within a context surrounding $C[i]$ that includes $C[i], K$ pre notes immediately preceding $C[i]$ and $K_{\text {post }}-1$ notes immediately following $C[i] . K_{\text {pre }}$ is called the precontext and $K_{\text {post }}$ is called the postcontext.

Step 2 For each $0 \leq i<|C|$ and each pitch class $0 \leq p \leq 11$, compute the number of diatonic steps $(\bmod 7), D(i, p)$, that there would be from the tonic to the pitch name of $C[i]$ if $p$ were the pitch class of the tonic at the point where $C[i]$ occurs. (Assume that the notes are spelt as they are in a harmonic chromatic scale whose tonic has pitch class $p[21$, p. 78]).

Step 3 For each $0 \leq i<|C|$ and each pitch class $0 \leq p \leq 11$, compute the value

$$
D^{\prime}(i, p)=(D(i, p)-D(0, p)) \bmod 7 .
$$

$D^{\prime}(i, p)$ gives the number of diatonic steps $(\bmod 7)$ from the pitch name of the first note (i.e., the note corresponding to $C[0]$ ) to the pitch name of $C[i]$ if the tonic pitch class is $p$.

\footnotetext{
${ }^{2}$ Patent pending [18-20]. Please contact the author at dave@titanmusic.com if you wish to use the algorithm. Permission will usually be granted for free use of the algorithm for non-commercial purposes.
} 
Step 4 For each $0 \leq i<|C|$ and each diatonic interval $0 \leq d \leq 6$, compute the set of tonic pitch classes,

$$
X(i, d)=\left\{p \mid D^{\prime}(i, p)=d\right\} .
$$

$X(i, d)$ contains the tonic pitch classes that would lead to the diatonic interval from the first note to $C[i]$ being $d$.

Step 5 For each $0 \leq i<|C|$ and each diatonic interval $0 \leq d \leq 6$, compute the sum, $N(i, d)$, of the values of $C N T(i, p)$ for all the tonic pitch classes $p \in X(i, d)$. That is,

$$
N(i, d)=\sum_{p \in X(i, d)} C N T(i, p) .
$$

Step 6 For each $0 \leq i<|C|$, compute $d_{\max }(i)$, the value of $d$ for which $N(i, d)$ is a maximum.

Step 7 Assign a letter name to the first note, $C[0]$. This can be done, for example, by using the table below

\begin{tabular}{|l|llllllllllll|}
\hline$C[0]$ & 0 & 1 & 2 & 3 & 4 & 5 & 6 & 7 & 8 & 9 & 10 & 11 \\
\hline Letter name of $C[0]$ & C & C & $\mathrm{D}$ & $\mathrm{E}$ & $\mathrm{E}$ & $\mathrm{F}$ & $\mathrm{F}$ & $\mathrm{G}$ & $\mathrm{A}$ & $\mathrm{A}$ & $\mathrm{B}$ & $\mathrm{B}$ \\
\hline
\end{tabular}

Step 8 For each $0 \leq i<|C|$, make the letter name of the note corresponding to $C[i], d_{\max }(i)$ steps above the letter name assigned to the note corresponding to $C[0]$.

The actual letter name assigned to the first note in Step 7 is not critical. It might make the difference, for example, between a piece being spelt in Ab rather than $\mathrm{G} \sharp$ but it would not change the pitch interval name of the interval between any pair of notes in the piece. Clearly, if a correctly notated piece is transposed up or down by a diminished second, the resulting piece will still be correctly notated - it will just be written in a different key. In general, of course, the key is chosen so as to minimise the number of sharps or flats in the key signature as this is supposed to make the score easier to read. For example, a piece notated in $\mathrm{C}$ major would generally be regarded as being easier to read than the same piece notated in $\mathrm{B} \sharp$ major.

Stage 2 of the algorithm corrects those instances in the output of Stage 1 where a neighbour note or passing note is erroneously predicted to have the same letter name as either the note preceding it or the note following it. That is, the second stage of the algorithm corrects errors like those shown in Fig. 6.

In the first step of Stage 1, the algorithm essentially counts how many times each pitch class occurs within some specified context surrounding a particular note. Krumhansl [22, pp. 66-75] showed that there is a high correlation between the frequency with which a pitch class occurs within a passage and its perceived tonal stability as measured experimentally [23]. This suggests that the value $C N T(i, p)$, calculated in the first step of Stage 1, gives an approximate measure of the perceived tonal stability of the pitch class $p$ at the point in the music where 


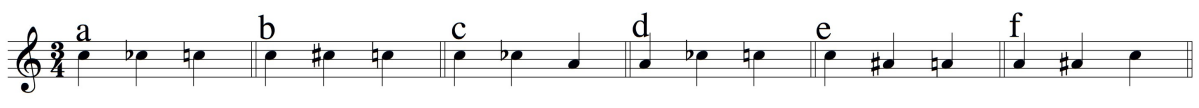

Fig. 6. Examples of the types of passing and neighbour note errors corrected in the second part of the ps13 algorithm

$C[i]$ occurs. In $p s 13$ the value $C N T(i, p)$ is used as a measure of the likelihood of $p$ being perceived to be the tonic at the point where note $C[i]$ occurs. Note that, unlike Temperley's algorithm, ps13 uses neither duration nor tempo and can deal with situations where two or more notes with the same pitch start at the same time.

\subsection{Results of Running ps13 on the First Book of J. S. Bach's Das Wohltemperirte Clavier}

In the first step of Stage 1, ps13 counts how many times each pitch class occurs within some specified context surrounding a particular note, defined by the values of $K_{\text {pre }}$ and $K_{\text {post }}$. In order to explore the effect that varying the values of $K_{\text {pre }}$ and $K_{\text {post }}$ has on the performance of ps13, the algorithm was run 2500 times on the test corpus, each time using a different pair of values for $K_{\text {pre }}$ and $K_{\text {post }}$, chosen so that both were between 1 and 50, inclusive. The image plot in Fig. 7 summarises the results obtained. It was found that ps13 made fewer than 122 mistakes (i.e., performed better than Temperley's algorithm) on the test corpus for $2004(80.160 \%)$ of the $2500\left\langle K_{\text {pre }}, K_{\text {post }}\right\rangle$ pairs tested.

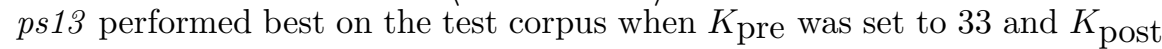
was set to either 23 or 25 . With these parameter values, ps13 made only 81 errors on the test corpus - that is, it correctly predicted the pitch names of $99.81 \%$ of the notes in the test corpus. The mean number of errors made by $p s 13$ over all $2500\left\langle K_{\text {pre }}, K_{\text {post }}\right\rangle$ pairs was 109.082 (i.e., $99.74 \%$ of the notes were correctly spelt on average over all $2500\left\langle K_{\text {pre }}, K_{\text {post }}\right\rangle$ pairs). This average value was better than the result obtained by Temperley's algorithm for this test corpus (see areas in Fig. 7 corresponding to scores greater than $99.71 \%$ ). The worst result was obtained when both $K_{\text {pre }}$ and $K_{\text {post }}$ were set to 1 . In this case, $p s 13$ made 1117 errors $(97.31 \%$ correct). However, provided $K$ pre was greater than about 13 and $K_{\text {post }}$ was greater than about 16, ps13 predicted the correct pitch name for over $99.71 \%$ of the notes in the test corpus.

\section{Comparing the Algorithms on a Larger Corpus}

\subsection{Structure of the Larger Test Corpus}

ps13 and the algorithms of Temperley, Cambouropoulos and Longuet-Higgins were then run on a much larger corpus containing 1729886 notes and consisting 

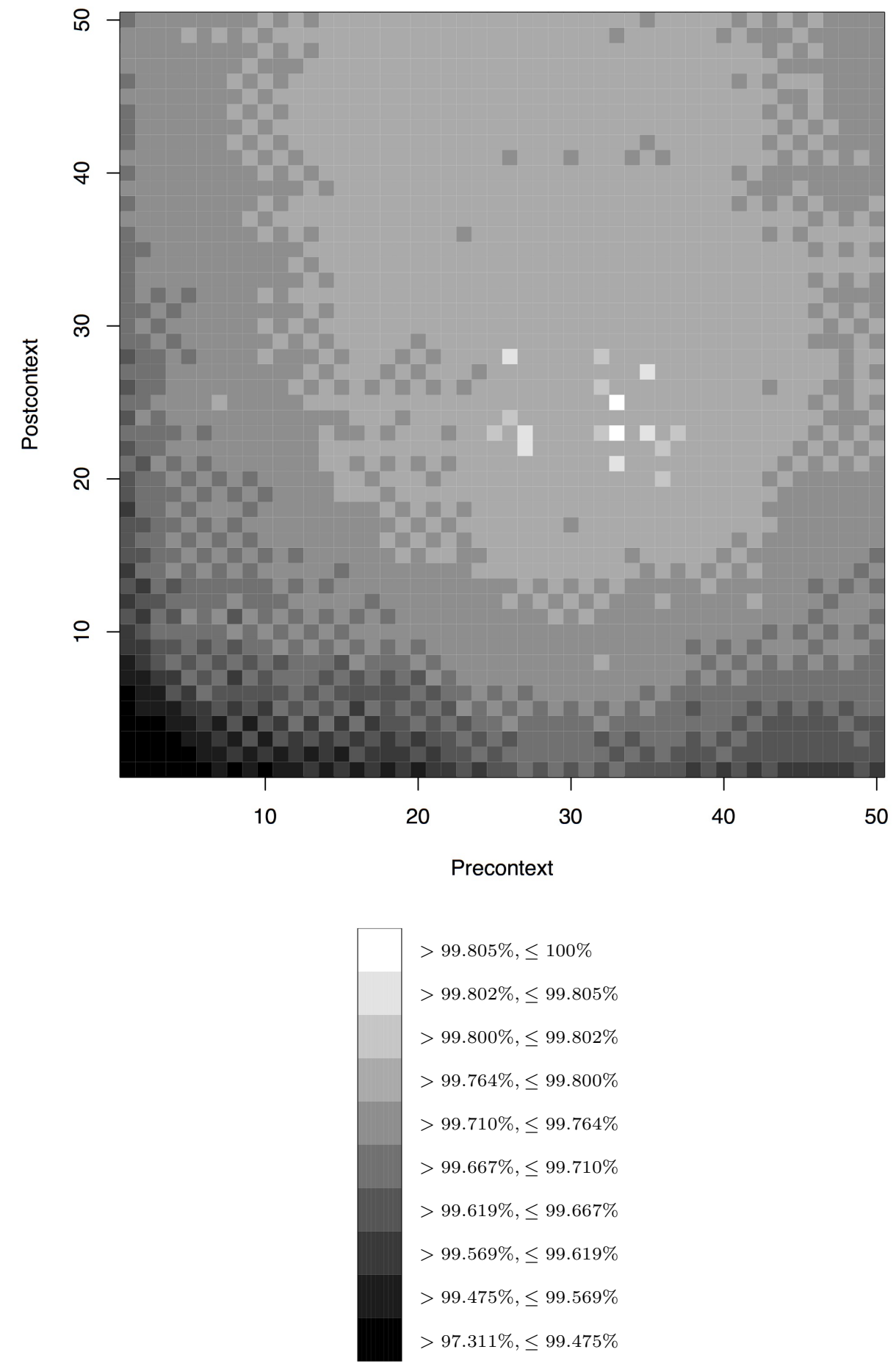

Fig. 7. Image plot showing percentage of notes spelt correctly by $p s 13$ for all values of $K$ pre and $K_{\text {post }}$ between 1 and 50 
of 1655 movements from works by 9 baroque and classical composers (Corelli, Vivaldi, Telemann, Bach, Handel, B. Marcello, Haydn, Mozart and Beethoven). The values of $K_{\text {pre }}$ and $K_{\text {post }}$ for ps13 were set to 33 and 23, respectively, these being the values that produced the best results when the algorithm was run on the smaller corpus in the pilot study. Table 2 shows the percentage and number of notes in this larger test corpus in works by each composer.

Table 2. Number and percentage of notes in large corpus in works by each composer

\begin{tabular}{|c|c|c|c|}
\hline Composer & Number of notes & \% of notes & Cum. \% \\
\hline Corelli & 31390 & $1.81 \%$ & $1.81 \%$ \\
Vivaldi & 223678 & $12.93 \%$ & $14.74 \%$ \\
Telemann & 89542 & $5.18 \%$ & $19.92 \%$ \\
Bach & 627083 & $36.25 \%$ & $56.17 \%$ \\
Handel & 449793 & $26.00 \%$ & $82.17 \%$ \\
Marcello & 2962 & $0.17 \%$ & $82.34 \%$ \\
Haydn & 84682 & $4.90 \%$ & $87.24 \%$ \\
Mozart & 172097 & $9.95 \%$ & $97.19 \%$ \\
Beethoven & 48659 & $2.81 \%$ & $100.00 \%$ \\
\hline Total & 1729886 & & \\
\hline
\end{tabular}

Table 2 reveals that more than $80 \%$ of the music in the corpus is baroque and the rest is classical. So the corpus is not very stylistically varied - it contains music written between about 1675 and 1825. Table 2 also shows that over $60 \%$ of the corpus consists of works by Bach and Handel. Indeed, the corpus is very unevenly distributed between the nine composers.

Unfortunately, a more varied and balanced corpus of a comparable size was not available at the time this experiment was carried out.

\subsection{Comparison of Algorithms with Respect to Number of Notes Spelt Correctly}

Table 3 shows the number of notes spelt incorrectly by each algorithm for each composer in the corpus. The bottom row in this table gives the total number of notes in the corpus spelt incorrectly by each algorithm.

Table 4 shows the percentage of notes spelt correctly by each algorithm for each composer. The bottom row of this table shows the total percentage of notes in the corpus spelt correctly by each algorithm. As shown in this table, overall, the percentage of notes spelt correctly is largest for the ps13 algorithm.

McNemar's test $[24,25]$ was then used to determine whether the differences between the scores achieved by the algorithms were statistically significant. ${ }^{3}$ McNemar's test is essentially a chi-squared test for related samples. Let's denote by $S_{A, T}$ and $S_{B, T}$ the percentages of notes spelt correctly by algorithms $A$ and $B$, respectively, when they are run on a test corpus $T$. Let us further assume that $S_{A, T}>S_{B, T}$. McNemar's test can be used to estimate the probability ( $p$-value)

\footnotetext{
${ }^{3}$ I am grateful to Marcus Pearce for suggesting the use of McNemar's test.
} 
Table 3. The number of notes spelt incorrectly by each algorithm for each composer

\begin{tabular}{|r|cccc|}
\hline & Cam & LH & ps13 & Tem \\
\hline Corelli & 148 & 120 & 30 & 6 \\
Vivaldi & 1816 & 5518 & 1497 & 4900 \\
Telemann & 604 & 665 & 481 & 111 \\
Bach & 13347 & 13022 & 3450 & 1166 \\
Handel & 1929 & 3342 & 2339 & 1309 \\
Marcello & 1 & 4 & 14 & 5 \\
Haydn & 1497 & 6174 & 823 & 6391 \\
Mozart & 2300 & 10147 & 2250 & 19832 \\
Beethoven & 600 & 1703 & 727 & 6525 \\
Complete test corpus & 22242 & 40695 & 11611 & 40245 \\
\hline
\end{tabular}

Table 4. Percentage of notes spelt correctly by each algorithm for each composer

\begin{tabular}{|r|cccc|}
\hline & Cam & LH & ps13 & Tem \\
\hline Corelli & $99.53 \%$ & $99.62 \%$ & $99.90 \%$ & $99.98 \%$ \\
Vivaldi & $99.19 \%$ & $97.53 \%$ & $99.33 \%$ & $97.81 \%$ \\
Telemann & $99.33 \%$ & $99.26 \%$ & $99.46 \%$ & $99.88 \%$ \\
Bach & $97.87 \%$ & $97.92 \%$ & $99.45 \%$ & $99.81 \%$ \\
Handel & $99.57 \%$ & $99.26 \%$ & $99.48 \%$ & $99.71 \%$ \\
Marcello & $99.97 \%$ & $99.86 \%$ & $99.53 \%$ & $99.83 \%$ \\
Haydn & $98.23 \%$ & $92.71 \%$ & $99.03 \%$ & $92.45 \%$ \\
Mozart & $98.66 \%$ & $94.10 \%$ & $98.69 \%$ & $88.48 \%$ \\
Beethoven & $98.77 \%$ & $96.50 \%$ & $98.51 \%$ & $86.59 \%$ \\
Complete test corpus & $98.71 \%$ & $97.65 \%$ & $99.33 \%$ & $97.67 \%$ \\
\hline
\end{tabular}

of getting results at least as different as $S_{A, T}$ and $S_{B, T}$ if there would actually be no difference between the percentage of notes spelt correctly by $A$ and $B$ if they were run on the population from which $T$ was taken. The first step in computing this $p$-value is to compute the values $n_{i c}$, the number of notes incorrectly spelt by $A$ but correctly spelt by $B$; and $n_{c i}$, the number of notes correctly spelt by $A$ but incorrectly spelt by $B$. If $A$ and $B$ would actually perform equally well on the whole population, then we expect $n_{c i}$ to be equal to $n_{i c}$. McNemar's test assumes that the expected values of $n_{c i}$ and $n_{i c}$ are both equal to $\left(n_{c i}+n_{i c}\right) / 2$ and compares the observed values of $n_{c i}$ and $n_{i c}$ with this expected value. An estimate of $\chi^{2}$ is then obtained using the following formula

$$
\chi^{2}=\frac{\left(\left|n_{i c}-n_{c i}\right|-1\right)^{2}}{n_{i c}+n_{c i}} .
$$

The results of this analysis are shown in Table 5 . In this table, each value in a column headed ' $p$-value' gives the statistical significance (expressed as a probability computed using McNemar's test) of the difference in performance between the algorithms to the left and right of the value in the table. For example, the value in the seventh column and first row of Table 5 indicates that if the algorithms of Longuet-Higgins and Cambouropoulos would actually make an equal number of note errors when run over the whole population of works represented by the test corpus, then the probability of getting a difference in scores at least as great as that observed in this study would be 0.0765 . The bottom row of this table shows that, overall, ps13 spelt very significantly more notes correctly 
than Cambouropoulos's algorithm $(p<0.0001)$, which in turn spelt very significantly more notes correctly than Temperley's algorithm $(p<0.0001)$. However, the percentage of notes spelt correctly by Temperley's algorithm (97.67\%) and Longuet-Higgins's algorithm $(97.65 \%)$ did not differ significantly $(p=0.0954)$. (A difference is considered significant if $p \leq 0.05$.)

Table 5. Statistical significance of differences between note error rates of algorithms for each composer and for complete corpus, measured using McNemar's test

\begin{tabular}{|r||c|c|c|c|c|c|c|}
\hline Composer & Best & $p$-value & 2nd best & $p$-value & 3rd best & $p$-value & Worst \\
\hline Corelli & Tem & $<0.0001$ & ps13 & $<0.0001$ & LH & 0.0765 & Cam \\
Vivaldi & ps13 & $<0.0001$ & Cam & $<0.0001$ & Tem & $<0.0001$ & LH \\
Telemann & Tem & $<0.0001$ & ps13 & $<0.0001$ & Cam & 0.0736 & LH \\
Bach & Tem & $<0.0001$ & ps13 & $<0.0001$ & LH & 0.0352 & Cam \\
Handel & Tem & $<0.0001$ & Cam & $<0.0001$ & ps13 & $<0.0001$ & LH \\
Marcello & Cam & 0.1797 & LH & 0.7388 & Tem & 0.0389 & ps13 \\
Haydn & ps13 & $<0.0001$ & Cam & $<0.0001$ & LH & 0.0348 & Tem \\
Mozart & ps13 & 0.3665 & Cam & $<0.0001$ & LH & $<0.0001$ & Tem \\
Beethoven & Cam & $<0.0001$ & ps13 & $<0.0001$ & LH & $<0.0001$ & Tem \\
Complete test corpus & ps13 & $<0.0001$ & Cam & $<0.0001$ & Tem & 0.0954 & LH \\
\hline
\end{tabular}

The graph in Fig. 8 shows the percentage of notes spelt correctly by each algorithm for each composer, the composers being placed along the horizontal axis in increasing chronological order of birth. This graph suggests that the algorithms of Temperley and Longuet-Higgins perform significantly worse on the classical composers (Haydn, Mozart and Beethoven) than they do on the baroque composers. The graph also suggests that ps13 performs more consistently across the different composers and styles than the other algorithms.

\subsection{Comparison of Algorithms with Respect to Number of Intervals Spelt Correctly}

When the lists of errors generated by the algorithms were examined, it was observed that, in some cases, many errors were the result of large segments of the music simply being transposed up or down by a diminished second. In other words, a single incorrect interval between two consecutive notes in the input representation resulted in a whole segment of notes following the incorrect interval being spelt incorrectly. The algorithms were therefore also compared with respect to the number of intervals spelt correctly between consecutive notes in the input representations. Table 6 shows the number of intervals between consecutive notes in works by each composer in the corpus.

Table 7 shows the percentage of intervals spelt correctly by each algorithm for each composer and Table 8 shows the number of intervals spelt incorrectly by each algorithm for each composer.

The graph in Fig. 9 shows the percentage of intervals between consecutive notes spelt correctly by each algorithm for each composer, the composers being placed along the horizontal axis in increasing chronological order of birth. From 


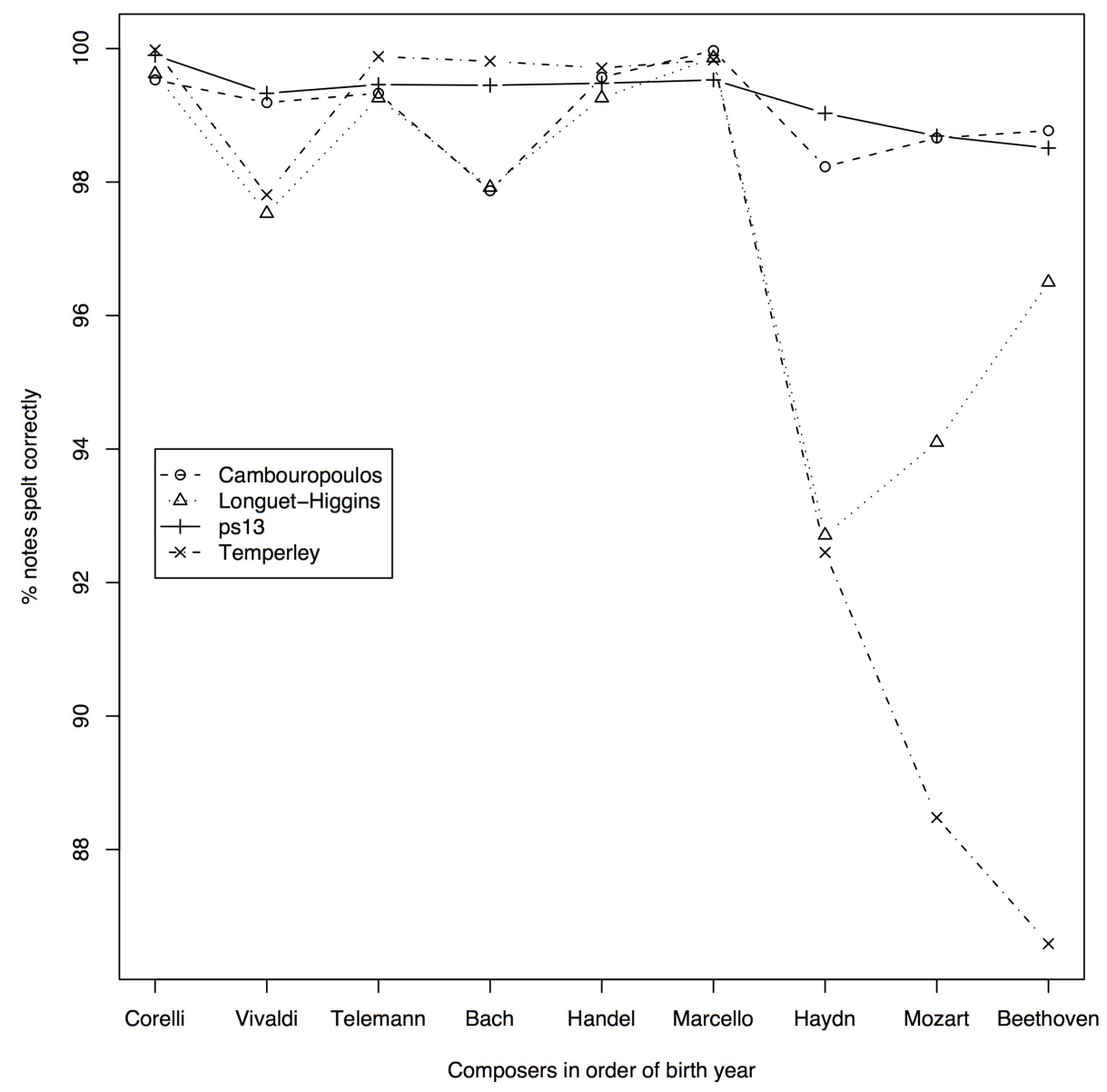

Fig. 8. Graph showing the percentage of notes spelt correctly by each algorithm for each composer, with the composers arranged along the horizontal axis in increasing chronological order of birth

Table 6. The number of intervals between consecutive notes in the input representations in works by each composer in the test corpus 
Table 7. Percentage of intervals between consecutive notes in the input representations spelt correctly by each algorithm for each composer

\begin{tabular}{|r|cccc|}
\hline & Cam & LH & ps13 & Tem \\
\hline Corelli & $99.08 \%$ & $99.71 \%$ & $99.81 \%$ & $99.96 \%$ \\
Vivaldi & $99.08 \%$ & $99.43 \%$ & $99.13 \%$ & $99.58 \%$ \\
Telemann & $99.19 \%$ & $99.50 \%$ & $99.12 \%$ & $99.77 \%$ \\
Bach & $97.97 \%$ & $99.07 \%$ & $99.28 \%$ & $99.67 \%$ \\
Handel & $99.44 \%$ & $99.62 \%$ & $99.55 \%$ & $99.82 \%$ \\
Marcello & $99.93 \%$ & $99.73 \%$ & $99.12 \%$ & $99.73 \%$ \\
Haydn & $97.93 \%$ & $97.99 \%$ & $98.47 \%$ & $98.13 \%$ \\
Mozart & $98.49 \%$ & $98.41 \%$ & $98.28 \%$ & $98.33 \%$ \\
Beethoven & $98.49 \%$ & $98.41 \%$ & $98.57 \%$ & $98.08 \%$ \\
Complete test corpus & $98.65 \%$ & $99.16 \%$ & $99.17 \%$ & $99.45 \%$ \\
\hline
\end{tabular}

Table 8. Number of intervals between consecutive notes in the input representations spelt incorrectly by each algorithm for each composer

\begin{tabular}{|r|cccc|}
\hline & Cam & LH & ps13 & Tem \\
\hline Corelli & 288 & 92 & 60 & 12 \\
Vivaldi & 2064 & 1264 & 1937 & 941 \\
Telemann & 727 & 450 & 788 & 204 \\
Bach & 12697 & 5852 & 4507 & 2074 \\
Handel & 2518 & 1703 & 2030 & 822 \\
Marcello & 2 & 8 & 26 & 8 \\
Haydn & 1750 & 1700 & 1298 & 1579 \\
Mozart & 2596 & 2734 & 2955 & 2874 \\
Beethoven & 736 & 772 & 695 & 933 \\
Complete test corpus & 23378 & 14575 & 14296 & 9447 \\
\hline
\end{tabular}

this graph it is clear that the algorithms of Longuet-Higgins and Temperley were better at spelling intervals between consecutive notes correctly than they were at spelling notes correctly. In particular, this graph shows that most of the note spelling errors made by Longuet-Higgins and Temperley's algorithms on the music of Haydn, Mozart and Beethoven were due to whole segments being spelt a diminished second away from the correct spelling.

Again, the statistical significance of the differences between the performances of the algorithms were analysed using McNemar's test and the results are shown in Table 9. The bottom line of this table reveals that, with respect to the number of intervals spelt correctly, Temperley's algorithm performs significantly better on this test corpus than the other three algorithms.

Nonetheless, the graph in Fig. 9 suggests that, even in terms of the number of intervals spelt correctly, all the algorithms perform worse on the classical music than on the baroque music.

\section{Conclusions and Further Work}

This paper presents the results of a study in which four pitch spelling algorithms were compared on a large corpus of tonal music. The algorithms compared were those of Cambouropoulos, Temperley and Longuet-Higgins, together with the author's own ps13 algorithm. When the algorithms were evaluated in terms 


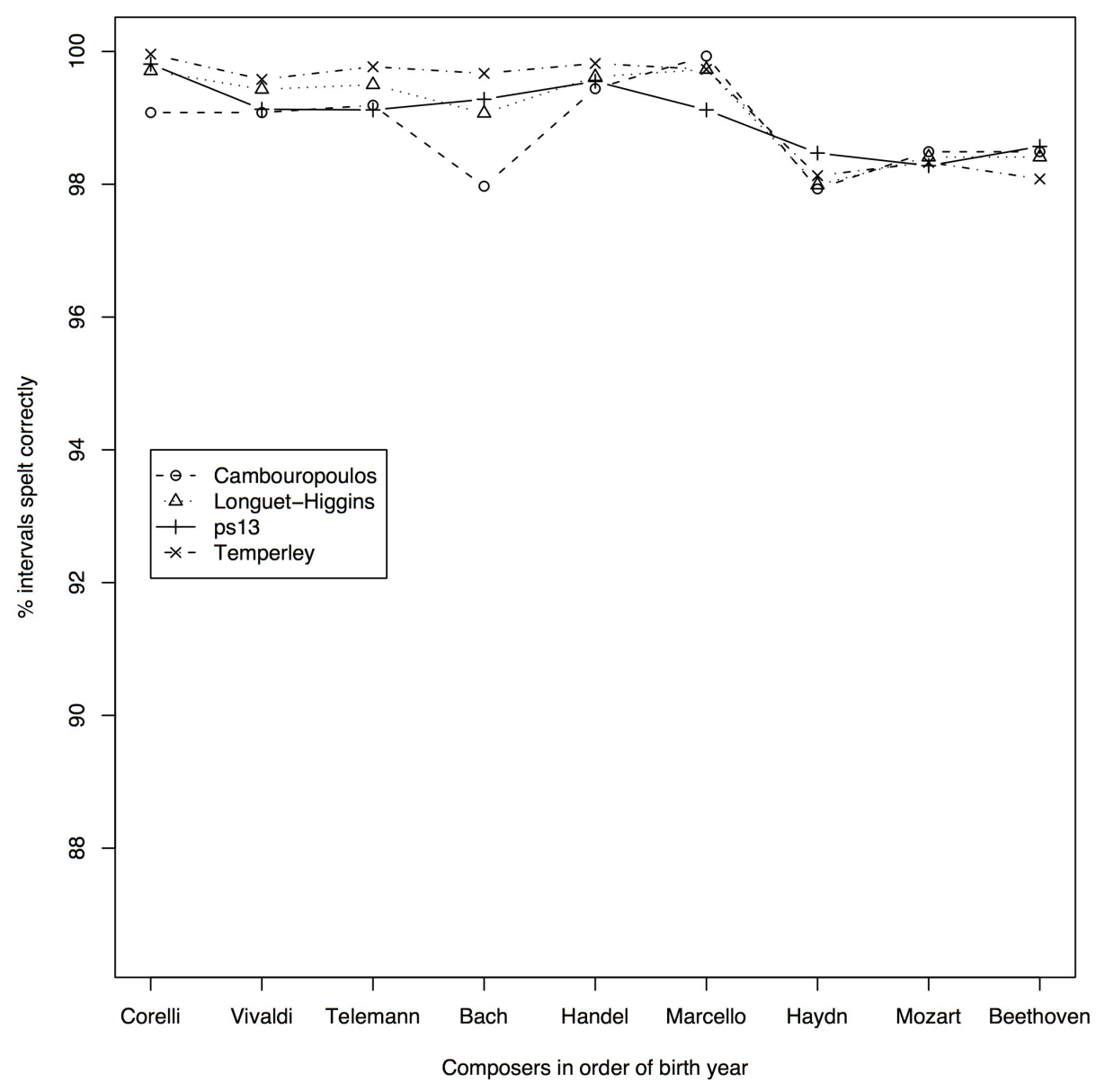

Fig. 9. Graph showing the percentage of intervals between consecutive notes spelt correctly by each algorithm for each composer, with the composers arranged along the horizontal axis in increasing chronological order of birth

Table 9. Statistical significance of differences between interval error rates of algorithms for each composer and for complete corpus, measured using McNemar's test

\begin{tabular}{|r|c|c|c|c|c|c|c|}
\hline Composer & Best & $p$-value & 2nd best & $p$-value & 3rd best & $p$-value & Worst \\
\hline Corelli & Tem & $<0.0001$ & ps13 & 0.0068 & LH & $<0.0001$ & Cam \\
Vivaldi & Tem & $<0.0001$ & LH & $<0.0001$ & ps13 & 0.1077 & Cam \\
Telemann & Tem & $<0.0001$ & LH & $<0.0001$ & Cam & 0.0029 & ps13 \\
Bach & Tem & $<0.0001$ & ps13 & $<0.0001$ & LH & $<0.0001$ & Cam \\
Handel & Tem & $<0.0001$ & LH & $<0.0001$ & ps13 & $<0.0001$ & Cam \\
Marcello & Cam & $0.0577,0.0577$ & LH,Tem & $0.001,0.002$ & ps13 & & \\
Haydn & ps13 & $<0.0001$ & Tem & 0.0028 & LH & 0.2416 & Cam \\
Mozart & Cam & 0.0584 & LH & 0.0143 & Tem & 0.2459 & ps13 \\
Beethoven & ps13 & 0.041 & Cam & 0.0252 & LH & $<0.0001$ & Tem \\
Complete test corpus & Tem & $<0.0001$ & ps13 & 0.0637 & LH & $<0.0001$ & Cam \\
\hline
\end{tabular}


of the number of notes in this corpus that they spelt correctly, it was found that the author's ps13 algorithm performed significantly better than the other algorithms, correctly spelling $99.33 \%$ of the notes in the corpus correctly. While all four algorithms performed well on the baroque music in the test corpus, it was found that the algorithms that were directly based on the circle of fifths (i.e., those of Temperley and Longuet-Higgins) performed less well than the other algorithms on the classical music in the corpus. However, when the algorithms were evaluated in terms of the number of intervals between consecutive notes spelt correctly, it was found that all the algorithms performed very well across all styles, with Temperley's algorithm performing best, correctly spelling $99.45 \%$ of the intervals in the corpus correctly.

It would be interesting to extend this study by doing further comparisons between the algorithms described here and other algorithms such as the real-time algorithms of Chew and Chen [26] and also the algorithms implemented in commercial music notation programs such as Sibelius (http://www.sibelius.com) and Finale (http://www.finalemusic.com). These algorithms should also be tested on other corpora containing works in a wider variety of tonal styles including, for example, romantic, impressionist, rock and jazz music.

Krumhansl [22, p. 79] claims that "once a key (or key region) has been determined, the correct spellings of the tones will be able to be determined in most cases". ps13 and the algorithms of Temperley and Longuet-Higgins all perform something at least very much like key finding as part of the pitch-spelling process. However, these algorithms give different results when run on the same test corpora. This demonstrates that there are various plausible ways of using the key-structure of a passage to determine pitch names and it is not at all obvious which of these methods will give the best results. Krumhansl's claim needs to be tested by building complete pitch spelling algorithms based on various key-finding algorithms and comparing the performance of these algorithms with others such as those described in this paper.

Finally, the errors made by the algorithms in the experiments described above need to be analysed in more depth in order to determine if any further improvements can be made.

\section{Acknowledgements}

While carrying out the research reported in this paper, the author was funded by the EPSRC (grant numbers GR/N08049/01 and GR/S17253/01). The author would like to thank Geraint Wiggins, Tim Crawford, Marcus Pearce and the other members of the ISMS group at City University, London (http://ccc.soi.city.ac.uk/isms/) for their kind and helpful suggestions and support.

\section{References}

1. Davy, M., Godsill, S.J.: Bayesian harmonic models for musical signal analysis (with discussion). In Bernardo, J.M., Berger, 
J.O., Dawid, A.P., Smith, A.F.M., eds.: Bayesian Statistics. Volume VII. Oxford University Press (2003) Draft available online at http://www-sigproc.eng.cam.ac.uk/ sjg/papers/02/harmonicfinal2.ps.

2. Walmsley, P.J.: Signal Separation of Musical Instruments. PhD thesis, Signal Processing Group, Department of Engineering, University of Cambridge (2000)

3. Plumbley, M., Abdallah, S., Bello, J., Davies, M.E., Monti, G., Sandler, M.: Automatic music transcription and audio source separation. Cybernetics and Systems 33 (2002) 603-627

4. Meredith, D., Lemström, K., Wiggins, G.A.: Algorithms for discovering repeated patterns in multidimensional representations of polyphonic music. Journal of New Music Research 31 (2002) 321-345 Draft available online at http://www.titanmusic.com/papers/public/siajnmr_submit_2.pdf.

5. Piston, W.: Harmony. Victor Gollancz Ltd., London (1978) Revised and expanded by Mark DeVoto.

6. Cambouropoulos, E.: A general pitch interval representation: Theory and applications. Journal of New Music Research 25 (1996) 231-251

7. Cambouropoulos, E.: Towards a General Computational Theory of Musical Structure. PhD thesis, University of Edinburgh (1998) Available online at http://users . auth.gr/ emilios/englishpage/phd.html.

8. Cambouropoulos, E.: Automatic pitch spelling: From numbers to sharps and flats. In: VIII Brazilian Symposium on Computer $\mathrm{Mu}$ sic (SBC\&M 2001), Fortaleza, Brazil (2001) Available online at ftp://ftp.ai.univie.ac.at/papers/oefai-tr-2001-12.pdf.

9. Cambouropoulos, E.: Pitch spelling: A computational model. Music Perception 20 (2003) 411-429

10. Longuet-Higgins, H.C.: The perception of melodies. Nature 263 (1976) 646-653

11. Longuet-Higgins, H.C.: The perception of melodies. In Longuet-Higgins, H.C., ed.: Mental Processes: Studies in Cognitive Science. British Psychological Society/MIT Press, London, England and Cambridge, Mass. (1987) 105-129

12. Longuet-Higgins, H.C.: The perception of melodies. In Schwanauer, S.M., Levitt, D.A., eds.: Machine Models of Music. M.I.T. Press, Cambridge, Mass. (1993) 471-495

13. Temperley, D.: An algorithm for harmonic analysis. Music Perception 15 (1997) $31-68$

14. Temperley, D.: The Cognition of Basic Musical Structures. MIT Press, Cambridge, MA (2001)

15. Meredith, D.: Pitch spelling algorithms. In Kopiez, R., Lehmann, A.C., Wolther, I., Wolf, C., eds.: Proceedings of the Fifth Triennial ESCOM Conference (ESCOM5) (8-13 September 2003), Hanover University of Music and Drama, Hanover, Germany. (2003) pp. 204-207 Draft available online at http://www.titanmusic.com/papers/public/ps13-escom-paper.pdf.

16. Hewlett, W.B.: MuseData: Multipurpose representation. In Selfridge-Field, E., ed.: Beyond MIDI: The Handbook of Musical Codes. MIT Press, Cambridge, MA. (1997) 402-447

17. Regener, E.: Pitch Notation and Equal Temperament: A Formal Study. University of California Press, Berkeley, CA. (1973)

18. Meredith, D.: Method of computing the pitch names of notes in MIDIlike music representations (2003) Patent filing submitted to UK Patent Office on 11 April 2003. Application number 0308456.3. Draft available online at http://www.titanmusic.com/papers/public/ps13-patent-1.pdf. 
19. Meredith, D.: Method of computing the pitch names of notes in MIDI-like music representations (2004) Patent filing submitted to UK Patent Office on 18 March 2004. Application number 0406166.9. Priority date 11 April 2003. Draft available online at http://www.titanmusic.com/papers/public/ps13-patent-2.pdf.

20. Meredith, D.: Method of computing the pitch names of notes in MIDI-like music representations (2004) Patent filing submitted to US Patent Office on 12 April 2004. Application number 10/821962. Priority date 11 April 2003. Draft available online at http://www.titanmusic.com/papers/public/us-ps13-patent.pdf.

21. Associated Board of the Royal Schools of Music: Rudiments and Theory of Music. Associated Board of the Royal Schools of Music, 14 Bedford Square, London, WC1B 3JG (1958)

22. Krumhansl, C.L.: Cognitive Foundations of Musical Pitch. Volume 17 of Oxford Psychology Series. Oxford University Press, New York and Oxford (1990)

23. Krumhansl, C.L., Kessler, E.J.: Tracing the dynamic changes in perceived tonal organisation in a spatial representation of musical keys. Psychological Review 89 (1982) 334-368

24. McNemar, Q.: Psychological Statistics. 4th edn. John Wiley and Sons, New York (1969)

25. Dietterich, T.G.: Approximate statistical tests for comparing supervised classification learning algorithms. Neural Computation 10 (1998) 1895-1924

26. Chew, E., Chen, Y.C.: Determining context-defining windows: Pitch spelling using the spiral array. In: Fourth International Conference on Music Information Retrieval (ISMIR 2003), Baltimore, MD. (2003) Available online at http://www-rcf.usc.edu/ ${ }^{\sim}$ echew/papers/ISMIR4/ecyc-ismir4.pdf. 